\title{
Correction to: Novel mutations in KMT2B offer pathophysiological insights on childhood-onset progressive dystonia
}

\author{
Hormos Salimi Dafsari ${ }^{1,2} \cdot$ Rosanne Sprute $^{1,2} \cdot$ Gilbert Wunderlich $^{3,4} \cdot$ Hülya-Sevcan Daimagüler ${ }^{1,2}$ - Ezgi Karaca ${ }^{5,6}$. \\ Adriana Contreras $^{1,2} \cdot$ Kerstin Becker $^{1,2} \cdot$ Mira Schulze-Rhonhof $^{1} \cdot$ Karl Kiening $^{7} \cdot$ Tülay Karakulak $^{5,6}$ - Manja Kloss ${ }^{8}$. \\ Annette Horn ${ }^{9} \cdot$ Amande Pauls $^{4} \cdot$ Peter Nürnberg $^{10} \cdot$ Janine Altmüller $^{10} \cdot$ Holger Thiele $^{10} \cdot$ Birgit Assmann $^{11}$. \\ Anne Koy ${ }^{1,3} \cdot$ Sebahattin Cirak $^{1,2,3}$
}

Published online: 6 August 2019

(c) The Author(s), under exclusive licence to The Japan Society of Human Genetics 2019

\section{Correction to: Journal of Human Genetics \\ https://doi.org/10.1038/s10038-019-0625-1}

The authors noticed nomenclature errors in the publication. The description of the identified variant for patient 2 was not appropriate according to the most recent HGVS nomenclature regulations. In patient 2 , the correct description of the variant in KMT2B shall be c.3602dupC, p.M1202Dfs*22. In the legend for Figure 3 there was a typographical error, it shall be Y2488 and in the labeling of the Figure 3B it shall be $\mathrm{C} 2488$, since the variant was correctly described in our paper as c.7463A $>$ G, p.Y2488C. These corrections do not alter the results and scientific interpretation as discussed in the paper.The authors apologize for the incompliance with the HGVS nomenclature regulations. The corrected Abstract, Figure 1, Figure 2, and Table 1 were updated and are as follows.

These authors contributed equally: Anne Koy, Sebahattin Cirak

The original article can be found online at https://doi.org/10.1038/ s10038-019-0625-1.

Sebahattin Cirak

sebahattin.cirak@uk-koeln.de

1 Department of Pediatrics, Faculty of Medicine and University Hospital Cologne, University of Cologne, Cologne, Germany

2 Center for Molecular Medicine Cologne (CMMC), Faculty of Medicine, University of Cologne, Cologne, Germany

3 Center for Rare Diseases, Faculty of Medicine and University Hospital Cologne, University of Cologne, Cologne, Germany

4 Department of Neurology, Faculty of Medicine and University Hospital Cologne, University of Cologne, Cologne, Germany

5 Izmir Biomedicine and Genome Center, Izmir, Turkey

\section{ABSTRACT}

Rapid progress has recently been made in the elucidation of the genetic basis of childhood-onset inherited generalized dystonia (IGD) due to the implementation of genomic sequencing methodologies. We identified four patients with childhood-onset IGD harboring novel disease-causing mutations in lysine-specific histone methyltransferase 2B gene $(K M T 2 B)$ by whole-exome sequencing. The main focus of this paper is to gain novel pathophysiological insights through understanding the molecular consequences of these mutations.

The disease course is mostly progressive, evolving from lower limbs into generalized dystonia, which could be associated with dysarthria, dysphonia, intellectual disability, orofacial dyskinesia, and sometimes distinct dysmorphic facial features. In two patients, motor performances improved after bilateral implantation of deep brain stimulation in the globus pallidus internus (GPi-DBS). Pharmacotherapy with trihexyphenidyl reduced dystonia in two patients.

We discovered three novel $K M T 2 B$ mutations. Our analyses revealed that the mutation in patient 1 (c.7463 A > G, p. Y2488C) is localized in the highly conserved FYRC domain of KMT2B. This mutation holds the potential to alter the inter-domain FYR interactions, which could lead to $K M T 2 B$

6 Izmir International Biomedicine and Genome Institute, Dokuz Eylül University, Izmir, Turkey

7 Department of Neurosurgery, University Hospital, Heidelberg, Germany

8 Department of Neurology, University Hospital, Heidelberg, Germany

9 Department of General Pediatrics and Neonatology, University Children's Hospital, Düsseldorf, Germany

10 Cologne Center for Genomics (CCG), Faculty of Medicine, University of Cologne, Cologne, Germany

11 Department of Neuropediatrics, University Children's Hospital, Heidelberg, Germany 
A.

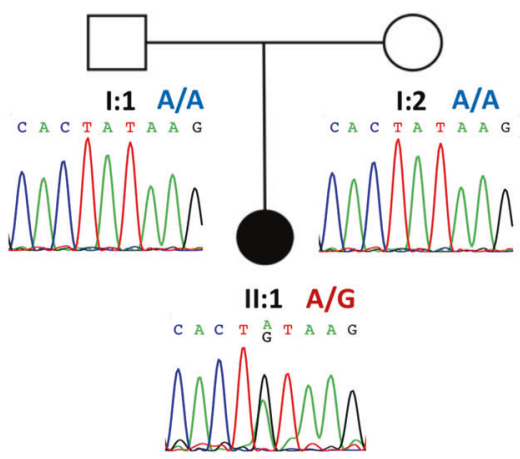

c.7463A>G, p.Y2488C
B.

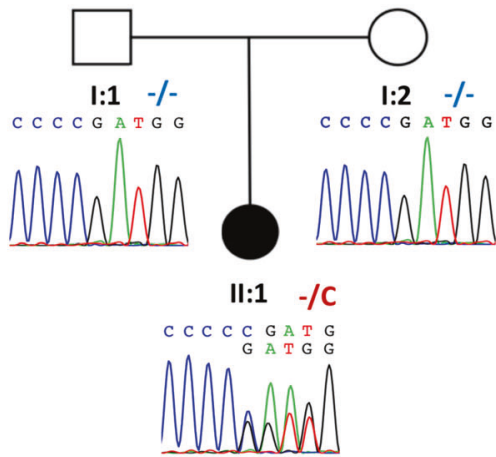

c.3602dupC, p.M1202Dfs*22
C.

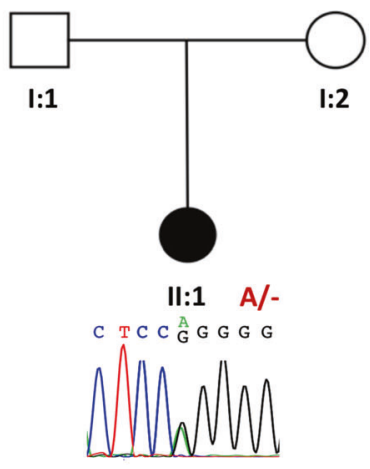

c.4229delA, p.Q1410Rfs*12
Fig. 1 Pedigree and chromatogram of the variants in $K M T 2 B$ in our three patients. a Pedigree and chromatogram of the variant in $K M T 2 B$ in patient 1 . The parents are healthy. The patient showed a single base exchange (c.7463A $>$ G, p.Y2488C). b Pedigree and chromatogram of the variant in $K M T 2 B$ in patient 2 . The parents are healthy. The patient showed a duplication and frame shift (c.3602dupC, p.M1202Dfs*22).
We show the strand as it is mutated in the patient (above) and also without the mutation (below) for comparison. c Pedigree and chromatogram of the variant in $K M T 2 B$ in patient 3 . The parents were not available for molecular genetic diagnoses. The patient showed a deletion (c.4229delA, p.Q1410Rfs*12)

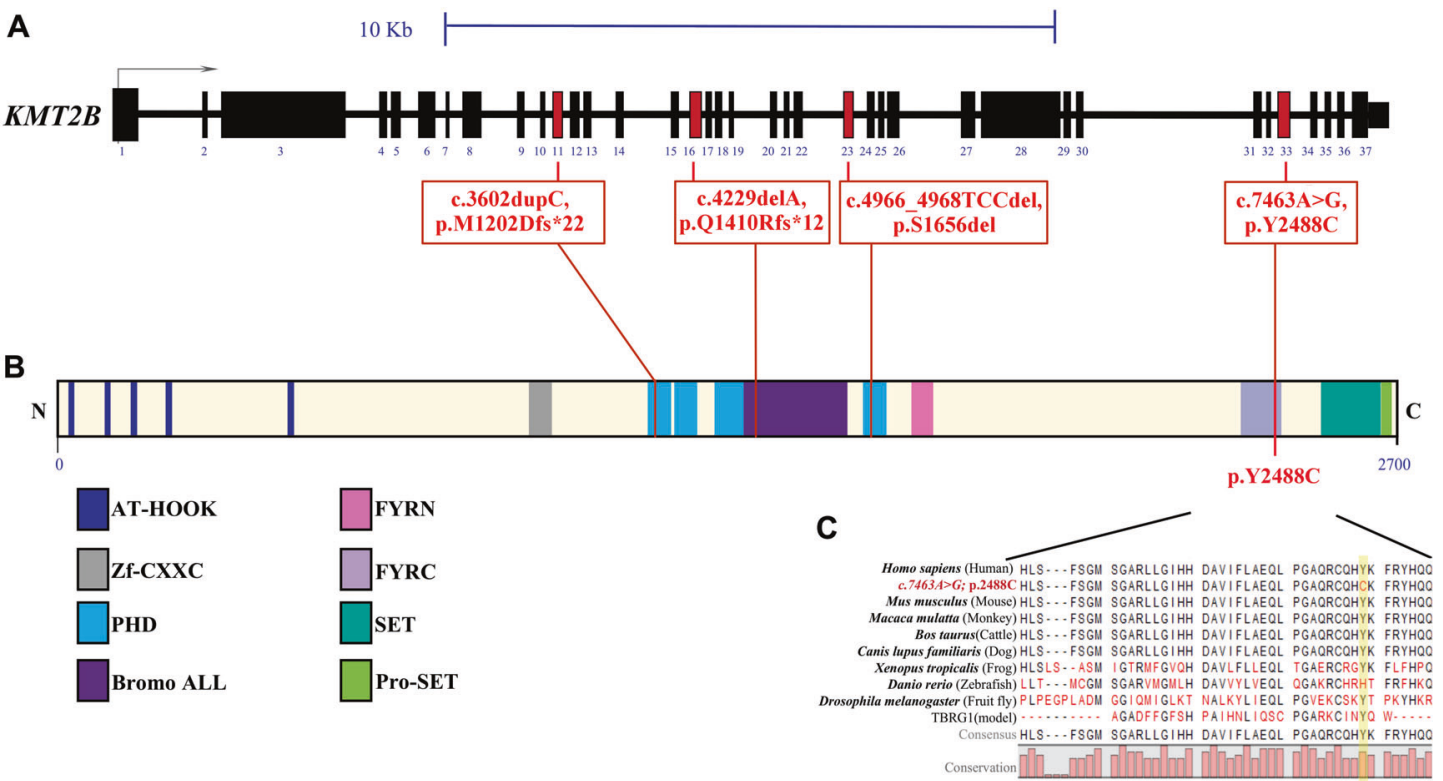

Fig. 2 Overview of $K M T 2 B$ variants and FYRC domain conservation between KMT2B homologs. a KMT2B locus. Gene structure (NM_014727.1) with 37 exons is represented. Variants found in our four patients are labeled in red. b KMT2B protein structure (Q9UMN6) with domains. Positions of frameshift or missense mutations are located in important protein domains, which are labeled in red. ATHook: DNA-binding motif consisting of a conserved, palindromic, core sequence of proline-arginine-glycine-arginine-proline binding to AT (adenosine, tyrosine). Zf-CXXC: domain containing eight conserved cysteine residues binding to two zinc ions. PHD: plant homeodomain (PHD) finger is a $\mathrm{C} 4 \mathrm{HC} 3$ zinc-finger-like motif found in nuclear proteins. Bromo ALL: an $~ 110$-amino-acid protein domain recognizing acetylated lysine residues like the $\mathrm{N}$-terminal tails of histones. FYRN and FYRC: "FY-rich" domain N-terminal (FYRN) and "FY-rich" domain C-terminal (FYRC) sequence motifs are two poorly characterized phenylalanine/tyrosine-rich regions of around 50 and 100 amino acids found in a variety of chromatin-associated proteins. SET: a 130-140 amino acid, evolutionary well-conserved sequence motif that was initially characterized in the Drosophila proteins $\mathrm{Su}(\mathrm{var}) 3-9$, Enhancer-of-zeste and Trithorax. Pro-SET: domain in the immediate vicinity to the SET domain. c Sequence alignment of top FYRC domains between KMT2B homologs, performed in CLC genomics workbench tool (protein accessions Human NP_055542.1; Mouse NP_001277502.1; Monkey XP_014979183.1; Cattle XP_015322911.1; Dog XP_003432729.3; Western Clawed Frog XP_002940678.2; Zebra Fish XP_009290151.1; Fruit Fly NP_599108.1; TBRG1 (model template NP_116200.2)) 


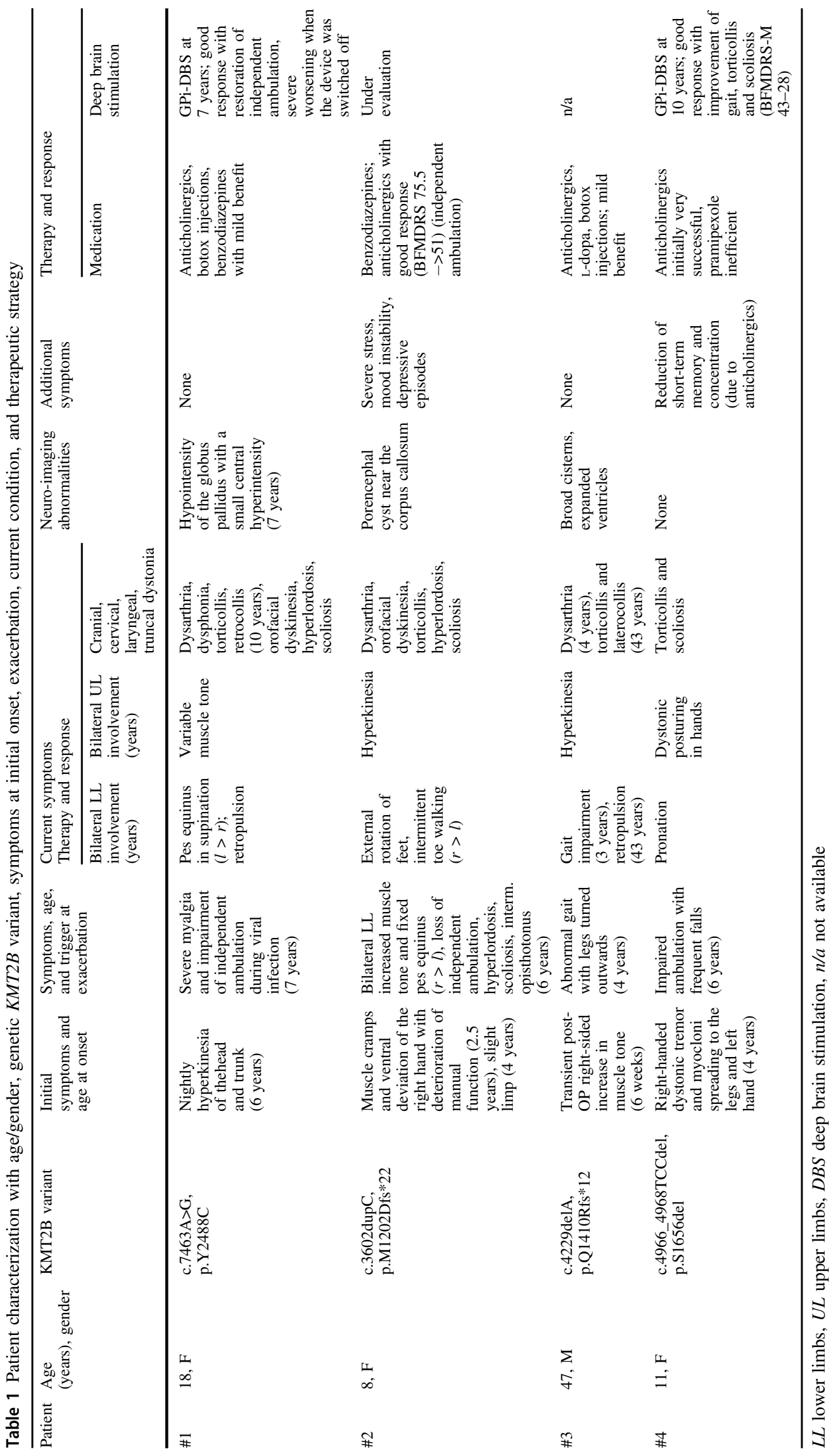


instability. The mutations in patients 2 and 3 (c.3602dupC, p.M1202Dfs*22; c.4229delA, p.Q1410Rfs*12) lead to predicted unstable transcripts, likely to be subject to degradation by non-sense-mediated decay.

Childhood-onset progressive dystonia with orofacial involvement is one of the main clinical manifestations of
KMT2B mutations. In all, $26 \%$ (18/69) of the reported cases have T2 signal alterations of the globus pallidus internus, mostly at a younger age. Anticholinergic medication and GPi-DBS are promising treatment options and shall be considered early (Figs. 1, 2 and Table 1). 